\title{
Compte rendu de Storibuk Pairundu. Tales and legends from the Kewa de Holger Jebens (ed.)
}

\section{Pascale Bonnemère}

\section{(2) OpenEdition \\ 1 Journals}

Édition électronique

URL : http://journals.openedition.org/jso/7863

DOI : $10.4000 /$ jso.7863

ISSN : $1760-7256$

Éditeur

Société des océanistes

\section{Édition imprimée}

Date de publication : 15 décembre 2017

Pagination : 364-366

ISSN : 0300-953x

\section{Référence électronique}

Pascale Bonnemère, "Compte rendu de Storibuk Pairundu. Tales and legends from the Kewa de Holger Jebens (ed.) », Journal de la Société des Océanistes [En ligne], 144-145| 2017, mis en ligne le 15 décembre 2017, consulté le 15 mars 2021. URL : http://journals.openedition.org/jso/7863 ; DOI : https://doi.org/10.4000/jso.7863

Ce document a été généré automatiquement le 15 mars 2021.

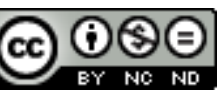

Journal de la société des océanistes est mis à disposition selon les termes de la Licence Creative Commons Attribution - Pas d'Utilisation Commerciale - Pas de Modification 4.0 International. 


\title{
Compte rendu de Storibuk Pairundu. Tales and legends from the Kewa de Holger Jebens (ed.)
}

\author{
Pascale Bonnemère
}

\section{RÉFÉRENCE}

JEBENS Holger (ed.), 2015. Storibuk Pairundu. Tales and legends from the Kewa (Southern Highlands, Papua New Guinea), collected by Alex Yapua Ari, Berlin, Reimer, 356 p., bibliogr., images.

1 Cet ouvrage rassemble plusieurs dizaines de récits (qualifiés de «tales and legends») recueillis dans le village kewa de Pairundu situé dans la province des Southern Highlands de Papouasie Nouvelle-Guinée. Les Kewa sont sans doute la population de ce pays dont la littérature orale est la mieux connue, l'anthropologue John LeRoy, parmi d'autres, y ayant déjà consacré deux volumes en 1985. Mais cette fois, c'est un jeune homme kewa, Alex Yapua Ari, qui prit l'initiative de transcrire dans un carnet les histoires que lui racontaient certains des habitants de son village, imitant en quelque sorte l'ethnologue Holger Jebens, qui s'était installé quelques semaines plus tôt à Pairundu (p. 3).

2 Le résultat est un livre de 350 pages contenant soixante-deux récits recueillis et transcrits en Tok Pisin (pidgin mélanésien) suivis de leur traduction en anglais et d'un court commentaire (une demi-page en moyenne) rédigé par H. Jebens. Celui-ci reprend ce que lui a dit Alex à propos du conteur et, le cas échéant, fournit l'interprétation du récit que le jeune homme fait lui-même et ajoute les références aux ouvrages de Beier (1977), Josephides (1982), MacDonald (1991) et LeRoy (1985a, 1985b) dans lesquels l'histoire figure également. Lorsque c'est nécessaire, il ajoute des éléments explicatifs sur la culture kewa dans des notes de bas de page incluses dans les récits. 
3 L'ouvrage comprend une courte introduction rédigée par Jebens dans laquelle il fournit le contexte de la collecte des récits et insiste sur les différences que ce livre présente par rapport aux autres traitant également de mythes kewa. Outre que les récits qu'il contient ont été rassemblés par un villageois et non par un ethnologue, ils sont ici mis en rapport avec ceux recueillis auparavant sur des thèmes similaires chez les Kewa. Enfin, l'ordre dans lequel ils sont présentés est celui d'Alex lui-même et n'émane donc pas d'un esprit extérieur qui les aurait organisés en fonction d'associations qu'il aurait lui-même établies.

4 Les thèmes abordés par les récits présentés dans cet ouvrage ne diffèrent guère de ceux rencontrés ailleurs en Nouvelle-Guinée : il y est question par exemple de relations entre germains, entre époux, de pénis coupés et parfois mangés par de vieilles femmes, d'oiseaux facétieux, des conséquences malheureuses de la transgression d'interdits, d'esprits des morts capturant des humains, de la transformation d'un être humain en un insecte ou un végétal - ou élément d'un végétal. Tous ces thèmes sont des motifs qui composent l'imaginaire de la population kewa de Pairundu.

5 Il n'est pas possible de s'attarder sur chacun de ces récits ni même sur une portion d'entre eux, qu'on les qualifie de mythes ou de contes et légendes comme l'ethnologue choisit de le faire ici (à la suite de LeRoy), mais il est intéressant de noter que, parmi ces soixante-deux histoires, plusieurs ont été inventées par Alex lui-même, ce qui nous rappelle que les récits oraux sont le produit de l'imaginaire d'une personne avant d'être le fruit d'une transmission et d'une appropriation collective. Les ethnologues oublient parfois cette dimension individuelle de la création tant les œuvres de toute société orale ont le plus souvent été analysées comme émanant d'une culture homogène. Les différentes versions d'un même mythe étaient alors considérées comme des distorsions liées aux conditions de la transmission orale et non comme des nuances nées de la volonté du narrateur d'imprimer sa marque.

6 On pourrait proposer qu'en distinguant dans leur langue les iti des rema, deux catégories de récits qualifiés par le terme de «stori» en Tok Pisin, les Kewa mettent l'accent sur cette différence entre les récits qui sont en quelque sorte cristallisés dans la culture - et dont font partie ce que la discipline a coutume d'appeler les mythes d'origine - et les histoires touchant moindrement aux thèmes fondamentaux de celle-ci et intégrant des éléments de la vie actuelle (avions, magasins, villes, etc.). En effet, les iti sont considérés comme des histoires provenant des ancêtres et transmises de génération en génération, alors que les rema parlent du présent ou d'un passé bien plus récent et d'événements qui sont considérés comme s'étant réellement passés (p. 3-4 et Franklin, 2017). À ces derniers, sont associés des noms de conteurs particuliers alors qu'il y a bien longtemps que l'on ne sait plus qui a, pour la première fois, raconté un iti particulier. La question de savoir si un rema est susceptible de devenir un iti au cours du temps est entière.

7 Jebens ne donne pas la raison pour laquelle les récits n'ont pas été recueillis en langue vernaculaire. Compte tenu du contexte particulier de leur collecte lors duquel des villageois racontent une histoire à l'un des leurs, une explication aurait été bienvenue. À priori, en effet, les iti et même les rema n'ont pas été transmis et inventés en Tok Pisin. Le lecteur se demande si l'idée vient d'Alex ou de l'ethnologue. Si elle émane du premier, le lecteur se demande quelle en serait la motivation. Elle ne peut en effet pas être celle de s'adresser à un large nombre de Papous puisque ceux qui lisent le Tok Pisin lisent aussi l'anglais'. Que le texte original ait été la transcription de la langue 
kewa que l'ethnologue aurait traduit en anglais n'aurait donc à cet égard rien changé. On peut imaginer qu'ainsi, la richesse de la langue aurait été préservée et que le livre aurait servi aux générations futures pour garder la mémoire de récits, sans passer par un processus de traduction dont on sait qu'il est susceptible de déformer l'original.

8 Cette légère critique mise à part et, bien qu'il n'apporte pas d'éléments d'analyse, Storibuk Pairundu est un maillon supplémentaire et bienvenu à la longue série des travaux sur les récits kewa à laquelle il faut ajouter l'ouvrage posthume de Bernard Juillerat paru en 2014, Le travail du mythe. La construction du héros en Mélanésie, dans la mesure où cet ethnologue y faisait référence à plusieurs de ceux collectés et analysés par John LeRoy.

\section{BIBLIOGRAPHIE}

BEIER Ulli (ed.), 1977. Kewa stories from the Southern Highlands collected by Stephen Rambi and Franck Nimi, Port Moresby, The Institute of Papua New Guinea Studies.

FRANKLIN Karl J., 2017. Two Kewa (Papua New Guinea) Story Genres, Language \& Linguistics in Melanesia 35, pp. 152-176.

JOSEPHIDES Lisette, 1982. Kewa stories and songs, Oral History 10 (2), pp. i-vi, 1-186.

JUILLERAT Bernard, 2014. Le travail du mythe. La construction du héros en Mélanésie, Paris, Société des Océanistes, Publications de la SdO 51.

LEROY John (ed.), 1985a. Kewa Tales, Vancouver, University of British Columbia Press.

LEROY John, 1985b. Fabricated World: An Interpretation of Kewa Tales, Vancouver, University of British Columbia Press.

MACDONALD Mary N., 1991. Mararoko: A Study in Melanesian Religion, New York, Peter Lang, American University Studies Series 11, Anthropology and Sociology 43.

\section{NOTES}

1. Dans le système éducatif papou en e et, l'anglais est très tôt la langue de l'apprentissage. 


\section{AUTEUR}

\section{PASCALE BONNEMÈRE}

CREDO, Marseille 